\title{
Spin response and topology of a staggered-Rashba superconductor
}

\author{
Anastasiia Skurativska $\odot,{ }^{1}$ Manfred Sigrist $\odot,{ }^{2}$ and Mark H. Fischer ${ }^{1}$ \\ ${ }^{1}$ Department of Physics, University of Zurich, Winterthurerstrasse 190, 8057 Zurich, Switzerland \\ ${ }^{2}$ Institute for Theoretical Physics, ETH Zurich, 8093 Zurich, Switzerland
}

(Received 18 March 2021; revised 21 July 2021; accepted 26 July 2021; published 9 August 2021)

\begin{abstract}
Inversion symmetry is a key symmetry in unconventional superconductors, and even its local breaking can have profound implications. For inversion-symmetric systems, there is a competition on a microscopic level between the spin-orbit coupling associated with the local lack of inversion and hybridizing terms that "restore" inversion. Investigating a layered system with alternating mirror-symmetry breaking, we study this competition considering the spin response of different superconducting order parameters for the case of strong spin-orbit coupling. We find that signatures of the local noncentrosymmetry, such as an increased spin susceptibility in spin-singlet superconductors for $T \rightarrow 0$, persist even into the quasi-three-dimensional regime. This leads to a direction-dependent spin response that allows us to distinguish different superconducting order parameters. Furthermore, we identify several regimes with possible topological superconducting phases within a symmetryindicator analysis. Our results may have direct relevance for the recently reported Ce-based superconductor $\mathrm{CeRh}_{2} \mathrm{As}_{2}$ and beyond.
\end{abstract}

DOI: 10.1103/PhysRevResearch.3.033133

\section{INTRODUCTION}

In superconductors with inversion symmetry, even- and odd-parity gap functions are distinguished by symmetry, and thus they correspond via the Pauli principle also to spin-singlet and spin-triplet pairing states, respectively. As a consequence, magnetic response can be used to distinguish the two cases. When the system lacks inversion, however, spin-singlet and spin-triplet states can mix [1]. In addition, the spin-orbit coupling associated with the broken symmetry-an example is the spin-orbit coupling of Rashba type for broken in-plane mirror symmetry - strongly restricts the possible spin-triplet order-parameter components, in other words it fixes the direction of the $d$ vector [2]. Moreover, even in the case of dominant spin-singlet or spin-triplet order parameters, the magnetic response, such as the spin susceptibility or critical fields, is not a feasible distinguishing probe anymore, as for spin-singlet superconductors a finite spin susceptibility for $T \rightarrow 0$ and unusually high critical fields can be expected. Beyond that, it was shown that topological properties of the phases, such as vortex bound states or surface flat bands, can serve as fingerprints of the respective phases [3,4].

Signatures of noncentrosymmetry can survive even in inversion-symmetric systems [5,6]. In particular, a crystal comprising weakly coupled sublattices whose subunits locally lack inversion, such as in the hexagonal SrPtAs [7] or even in some high-temperature cuprates [8], can exhibit an un-

Published by the American Physical Society under the terms of the Creative Commons Attribution 4.0 International license. Further distribution of this work must maintain attribution to the author(s) and the published article's title, journal citation, and DOI. conventional magnetic response or intriguing spin textures. In addition to specific crystal structures, this local noncentrosymmetricity can arise in artificial superlattices such as the regular stacks of superconducting $\mathrm{CeCoIn}_{5}$ alternating with layers of $\mathrm{YbCoIn}_{5}$ [9-11]. Note that the effect of local noncentrosymmetricity depends on the relative strength of inversion-breaking-induced spin-orbit coupling and intersublattice hybridization. The focus of most studies has thus been on quasi-two-dimensional systems with weak $c$-axis dispersion.

The recently discovered heavy-fermion superconductor $\mathrm{CeRh}_{2} \mathrm{As}_{2}$ with its tetragonal crystal structure belongs also to the class of locally noncentrosymmetric superconductors. In particular, it consists of layers with alternating inversionsymmetry breaking. The upper critical field directed along the $c$ axis (perpendicular to the staggered layers) extrapolates to $\sim 14 \mathrm{~T}$ at zero temperature, which lies far beyond the paramagnetic limiting field $H_{p} \sim 0.5 \mathrm{~T}$ for a critical temperature $T_{c} \approx 0.26 \mathrm{~K}$ [12]. Furthermore, the upper critical field shows a pronounced kink for a field $H \approx 4 \mathrm{~T}$. This anomaly strongly suggests a change in the order-parameter symmetry upon increasing magnetic field as further suggested in recent theoretical studies $[13,14]$. Note that the critical field for in-plane directions, on the other hand, extrapolates to only approximately $2 \mathrm{~T}$.

Unlike most staggered systems studied so far, $\mathrm{CeRh}_{2} \mathrm{As}_{2}$ is expected to have a rather strong $c$-axis dispersion, in other words it is a three-dimensional (3D) system. Being a Ce-based superconductor, we expect also a sizable spinorbit coupling. Motivated by these observations, we revisit the physics of locally noncentrosymmetric superconductors in situations where both the intersublattice hopping and the spin-orbit coupling strength are comparable to each other and the overall bandwidth. 


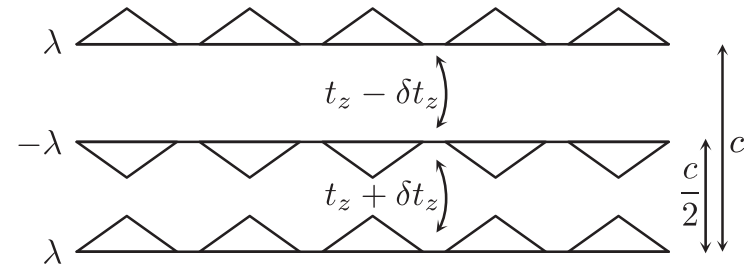

FIG. 1. Schematic of the system with the triangles indicating the mirror symmetry breaking. Note that, for simplicity, we choose the layers to be spaced equidistantly in the $z$ direction with unit-cell size $c$.

In particular, we investigate in detail a microscopic model of a layered system, where mirror symmetry is broken in a staggered fashion. By design, our model allows us to investigate how the system evolves from the 2D limit to the truly $3 \mathrm{D}$ case. For this purpose, we first study the spin susceptibility in the normal state, which leads us to identify four distinct regions, going from quasi-two-dimensional (q2D) all the way to truly 3D. Then we discuss possible order parameters and analyze their spin response, showing how both in-plane and out-of-plane fields are necessary to distinguish them. Eventually, we address the topological phases in the fully gapped case. Note that since the system retains inversion, we can use the recently developed concept of symmetry indicators [15-19] to show that the system can realize both first- and second-order topological superconducting phases.

\section{NORMAL-STATE PROPERTIES}

\section{A. Microscopic model}

We consider in the following a system of stacked layers, where each layer lacks a mirror symmetry in such a way that the resulting Rashba spin-orbit coupling alternates in sign; see Fig. 1. Such a system has centers of inversion, which lie in between two neighboring layers. However, electrons moving within an individual layer are subject to a spin-orbit coupling of Rashba type. For concreteness, we choose these layers to consist of a square lattice with point group symmetry $C_{4 v}$, while the full structure involves the tetragonal point group $D_{4 h}$. On a single layer, the electrons are governed by the Hamiltonian

$$
\mathcal{H}_{\mathrm{sl}}=\sum_{\mathbf{k} s} \xi_{\mathbf{k}}^{+} c_{\mathbf{k} s}^{\dagger} c_{\mathbf{k} s}+\sum_{\mathbf{k} s s^{\prime}} \vec{f}_{\mathbf{k}} \cdot \vec{\sigma}_{s s^{\prime}} c_{\mathbf{k} s}^{\dagger} c_{\mathbf{k} s^{\prime}},
$$

where $c_{\mathbf{k} s}^{\dagger}\left(c_{\mathbf{k} s}\right)$ creates (annihilates) an electron with momentum $\mathbf{k}$ and $\operatorname{spin} s$. Setting the in-plane lattice constant $a=1$,

$$
\xi_{\mathbf{k}}^{+}=-2 t\left(\cos k_{x}+\cos k_{y}\right)
$$

describes the dispersion due to nearest-neighbor hopping,

$$
\begin{aligned}
f_{\mathbf{k}}^{x} & =\lambda \sin k_{y}, \\
f_{\mathbf{k}}^{y} & =-\lambda \sin k_{x}
\end{aligned}
$$

enter the expression for the Rashba spin-orbit coupling, and $\vec{\sigma}$ denote the Pauli matrices.

The three-dimensional system with a staggered stacking of such layers, as shown in Fig. 1, is then described by the
Hamiltonian

$$
\mathcal{H}=\sum_{\mathbf{k}} \psi_{\mathbf{k} s}^{\dagger} \mathcal{H}_{\mathbf{k}} \psi_{\mathbf{k} s},
$$

with the $4 \times 4$ matrix

$$
\mathcal{H}_{\mathbf{k}}=\xi_{\mathbf{k}}^{+} \sigma_{0} \tau_{0}+\vec{\xi}_{\mathbf{k}}^{-} \cdot \sigma_{0} \vec{\tau}+\vec{f}_{\mathbf{k}} \cdot \vec{\sigma} \tau_{3},
$$

where the interlayer hopping is given by

$$
\begin{gathered}
\left(\xi_{\mathbf{k}}^{-}\right)_{1}=-2 t_{z} \cos \left(k_{z} / 2\right), \\
\left(\xi_{\mathbf{k}}^{-}\right)_{2}=-2 \delta t_{z} \sin \left(k_{z} / 2\right),
\end{gathered}
$$

and $\left(\xi_{\mathbf{k}}^{-}\right)_{3}=0$. Note that we have set the $z$-axis lattice constant $c=1$. Furthermore, we have introduced the Pauli matrices $\tau_{i}, i=1,2,3$, acting on the sublattice space of layers for the operators $\psi_{\mathbf{k} s}^{\dagger}=\left(c_{\mathrm{A} \mathbf{k} s}^{\dagger}, c_{\mathrm{Bk} s}^{\dagger}\right)$, which we denote by an $\mathrm{A}$ or B sublattice index.

The eigenenergies of this Hamiltonian are doubly degenerate and are given by

$$
\xi_{\mathbf{k} \pm}=\xi_{\mathbf{k}}^{+} \pm \sqrt{\left|\vec{\xi}_{\mathbf{k}}^{-}\right|^{2}+\left|\vec{f}_{\mathbf{k}}\right|^{2}}-\mu
$$

which we denote in the following as $\xi_{\alpha}$ with $\alpha= \pm$ neglecting the momentum index for shorter notation. Further, we introduce the chemical potential $\mu$. For concreteness, we use in the following $\lambda=0.5 t$ with $t$ the energy unit, and we choose $\mu$ so as to fix the density of electrons to $n_{\text {tot }}=0.15$ per unit cell. This choice of filling allows us to access different Fermi surface topologies ranging from quasi-2D to bulklike 3D; see Appendix A.

\section{B. Magnetic response}

It is instructive to first consider the normal-state magnetic response of this system. For this purpose, we introduce the normal-state Green's function, which is defined through

$$
G_{0}\left(\mathbf{k}, \omega_{n}\right)^{-1}=i \omega_{n} \sigma_{0} \tau_{0}-\mathcal{H}_{\mathbf{k}},
$$

with $\omega_{n}=(2 n+1) \pi T$ the fermionic Matsubara frequencies, with $n \in \mathbb{Z}$ and $T$ the temperature. This $4 \times 4$ Green'sfunction matrix can explicitly be calculated by inverting Eq. (10),

$$
G_{0}\left(\mathbf{k}, \omega_{n}\right)=G_{+}^{0} \sigma_{0} \tau_{0}+G_{-}^{0}\left(\hat{\xi}_{\mathbf{k}}^{-} \cdot \sigma_{0} \vec{\tau}+\hat{f}_{\mathbf{k}} \cdot \vec{\sigma} \tau_{3}\right),
$$

where we introduced

$$
\begin{aligned}
G_{ \pm}^{0} \equiv G_{ \pm}^{0}\left(\mathbf{k}, \omega_{n}\right) & =\frac{1}{2}\left(\frac{1}{i \omega_{n}-\xi_{+}} \pm \frac{1}{i \omega_{n}-\xi_{-}}\right) \\
\hat{\xi}_{\mathbf{k}}^{-} & =\frac{\vec{\xi}_{\mathbf{k}}^{-}}{\sqrt{\left|\vec{f}_{\mathbf{k}}\right|^{2}+\left|\vec{\xi}_{\mathbf{k}}^{-}\right|^{2}}},
\end{aligned}
$$

and

$$
\hat{f}_{\mathbf{k}}=\frac{\vec{f}_{\mathbf{k}}}{\sqrt{\left|\vec{f}_{\mathbf{k}}\right|^{2}+\left|\vec{\xi}_{\mathbf{k}}^{-}\right|^{2}}}
$$

To investigate the system's response to a magnetic field, we calculate the (normal-state) uniform, static spin susceptibility 
$(\mathbf{q}=\mathbf{0}, \omega=0)$, which reads

$$
\chi_{i j}^{0}=-\mu_{B}^{2} T \sum_{n} \sum_{\mathbf{k}} \operatorname{tr}\left[\sigma_{i} \tau_{0} G_{0}\left(\mathbf{k}, \omega_{n}\right) \sigma_{j} \tau_{0} G_{0}\left(\mathbf{k}, \omega_{n}\right)\right],
$$

where the trace runs over spin and layer indices. Performing the trace first, we find that due to spin-orbit coupling, the susceptibility has a generic form with two contributions: the first is

$$
\begin{aligned}
\chi_{\mathrm{P}}^{0}(\mathbf{k}) & =-4 \mu_{B}^{2} T \sum_{\omega_{n}}\left[\left(G_{+}^{0}\right)^{2}+\left(G_{-}^{0}\right)^{2}\right] \\
& =2 \mu_{B}^{2}\left[\frac{\partial n_{\mathrm{F}}\left(\xi_{+}\right)}{\partial \xi_{+}}+\frac{\partial n_{\mathrm{F}}\left(\xi_{-}\right)}{\partial \xi_{-}}\right] \propto S_{\mathbf{k}}(\mu)
\end{aligned}
$$

with $n_{\mathrm{F}}(\xi)$ the Fermi distribution. This term corresponds to the Pauli-like susceptibility. In other words, it is proportional to the spectral density at the Fermi level, $S_{\mathbf{k}}(\mu)$, and after the $k$-integration, the density of states of the two bands at the Fermi level, $N(\mu)$. The second is

$$
\begin{aligned}
\chi_{\mathrm{vV}}^{0}(\mathbf{k}) & =-4 \mu_{B}^{2} T \sum_{\omega_{n}}\left[\left(G_{+}^{0}\right)^{2}-\left(G_{-}^{0}\right)^{2}\right] \\
& =2 \mu_{B}^{2} \frac{n_{\mathrm{F}}\left(\xi_{+}\right)-n_{\mathrm{F}}\left(\xi_{-}\right)}{\sqrt{\left|\vec{f}_{\mathbf{k}}\right|^{2}+\left|\vec{\xi}_{\mathbf{k}}^{-}\right|^{2}}}
\end{aligned}
$$

and it originates from interband processes due to the spinorbit coupling, thus we refer to it as a van Vleck term. The susceptibility is a combination of these Pauli and van Vleck contributions, in particular

$$
\chi_{z}^{0}=\sum_{\mathbf{k}}\left|\hat{\xi}_{\mathbf{k}}^{-}\right|^{2} \chi_{\mathrm{P}}^{0}(\mathbf{k})+\left|\hat{f}_{\mathbf{k}}\right|^{2} \chi_{\mathrm{vV}}^{0}(\mathbf{k})
$$

for fields in the $z$ direction, $i=j=z$. For fields along the $x$ direction, we find

$$
\chi_{x}^{0}=\sum_{\mathbf{k}}\left[\left|\hat{\xi}_{\mathbf{k}}^{-}\right|^{2}+\left(\hat{f}_{\mathbf{k}}^{x}\right)^{2}\right] \chi_{\mathrm{P}}^{0}(\mathbf{k})+\left(\hat{f}_{\mathbf{k}}^{y}\right)^{2} \chi_{\mathrm{vV}}^{0}(\mathbf{k})
$$

and analogously for fields in the $y$ direction. This result generalizes the pure Rashba case $[1,20]$, which is recovered when setting $t_{z}=\delta t_{z}=0$. In the other limit, $t_{z}, \delta t_{z} \gg \lambda$, the weight of the van Vleck term is strongly reduced and the Pauli susceptibility dominates. Consequently, we can use the ratio of the van Vleck susceptibility to the total susceptibility for fields along the $z$ axis as a measure of the behavior related to (local) inversion-symmetry breaking in the system.

Figure 2 shows the ratio of the van Vleck susceptibility to the total susceptibility of the normal state as a function of the interlayer hopping $t_{z}$. The susceptibility shows a distinct behavior depending on the Fermi surface topology as indicated by the insets: In the quasi-two-dimensional (q2D) regime, where both bands are partially filled and with open Fermi surfaces, the van Vleck contribution drops rapidly with increasing $t_{z}$. Then, in the first quasi-3D regime (q3DI) with one closed Fermi surface, $\chi_{\mathrm{vV}}^{0} / \chi^{0}$ decreases essentially linearly, before dropping again more rapidly once the closed Fermi surface disappears in the second quasi-3D regime (q3DII). Finally, the remaining Fermi surface closes, at which point the full system is $3 \mathrm{D}$ and behaves completely centrosymmetric. Note that the cusps in the relative susceptibility are related to the Lifshitz transitions connecting different regimes (see

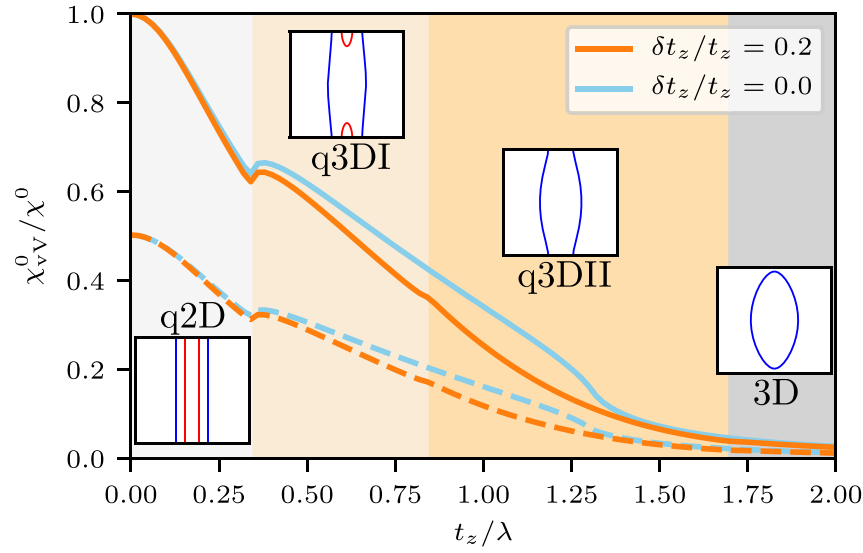

FIG. 2. Ratio of the van Vleck contribution to the total susceptibility in the normal state for fields along the $z$ direction (solid lines) and in-plane (dashed lines). For the case of $\delta t_{z} / t_{z}=0.2$, four different regimes are indicated, which are connected to the Fermi surface topology. The Fermi surfaces are illustrated by the insets, which show the Fermi surfaces in the $k_{x}-k_{z}$ plane of the Brillouin zone. For numerical reasons, we set $T=0.0025 t$.

Appendix A). As we will discuss in Sec. IV, different Fermisurface topologies are also connected to different possible topological phases for a triplet superconductor.

Before we move to calculating the spin susceptibility in the superconducting state, we can use the above finding to discuss what we expect for a spin-singlet gap function. As the Pauli term depends on the density of states at the Fermi level, which vanishes for a full (spin-singlet) gap, the corresponding susceptibility is expected to vanish as well. The van Vleck term, however, should stay constant, at least for $\sqrt{\left|\vec{f}_{\mathbf{k}}\right|^{2}+\left|\vec{\xi}_{\mathbf{k}}^{-}\right|^{2}} \gg$ $\Delta$, with $\Delta$ the superconducting gap. Comparing Eqs. (18) and (19), we further expect the susceptibility for fields in-plane to be half the size of the susceptibility for fields along $z$ for $T \rightarrow 0$, independent of the microscopic details, as long as the system has $C_{4}$ symmetry.

\section{SPIN RESPONSE OF THE SUPERCONDUCTOR}

\section{A. Superconducting order parameters}

Before calculating the spin response of possible superconducting phases, we discuss the possible pairing states of interest here. While mixing of spin-singlet and spin-triplet order parameters is allowed within the plane and microscopically supported by the Rashba spin-orbit coupling, the order parameter can still be classified as even or odd due to the global inversion symmetry [5]. Similar to the noncentrosymmetric situation $[1,2,20]$, most spin-triplet order parameters are suppressed by the spin-orbit coupling in the staggered case, too [5]. The energetically most stable gap structures correspond to intraband pairing, in other words to gap functions that are diagonal in the bands with energies given by Eq. (9) [21-23]. As these order parameters can also be treated analytically, we will focus exclusively on them. As a consequence, the order parameters we study have no in-plane mixing of spin-singlet and spin-triplet channels, which, to first order, will not influence their qualitative behavior. Note that 
in the following we assume the superconducting gap to be the smallest energy scale in the problem.

For this intraband pairing, we focus on the most symmetric gap functions leading to three different gap structures: a spinsinglet that transforms like $A_{1 g}$, a spin-triplet order parameter with its $d$-vector in-plane that transforms like $A_{2 u}$, and a spin-triplet order parameter with the corresponding $d$-vector along the $z$ axis that transforms like $A_{1 u}$ in $D_{4 h}$ [5]. We briefly discuss in the following all three order parameters.

For the spin-singlet channel, the intraband order parameter has the form

$$
\Delta^{\mathrm{s}}(\mathbf{k})=\psi(\mathbf{k}) i \sigma_{y} \tau_{0}
$$

This order parameter describes fully gapped $s$-wave spinsinglet pairing with $\psi(-\mathbf{k})=\psi(\mathbf{k}) \equiv \psi$.

For the spin-triplet gap, Cooper pairing can be both within the plane and between neighboring planes: The first,

$$
\Delta^{\|}(\mathbf{k})=\left(\vec{d}_{\mathbf{k}}^{\|} \cdot \vec{\sigma}\right) i \sigma_{y} \tau_{0}
$$

where $\vec{d}_{\mathbf{k}}^{\|}=\left(d_{\mathbf{k}}^{x}, d_{\mathbf{k}}^{y}, 0\right)^{\mathrm{T}} \| \vec{f}_{\mathbf{k}}$, is similar to the case of noncentrosymmetric superconductors $[1,20]$ and transforms with $A_{2 u}$. This gap vanishes for $k_{x}=k_{y}=0$, such that for closed Fermi surfaces as found in the q3DI and 3D regime, the gap has point nodes. Notice that in the noncentrosymmetric case with point group $C_{4 v}$, the gap functions of $A_{1 g}$ and $A_{2 u}$ symmetry mix, resulting instead in line nodes for closed Fermi surfaces and dominant triplet contribution.

In addition, the $A_{1 u}$ order parameter with out-of-plane spintriplet pairing is allowed due to the staggered nature of the mirror-symmetry breaking with a nearest-neighbor gap function

$$
\Delta^{\perp}(\mathbf{k})=\left[\left(\vec{d}_{\mathbf{k}}^{\perp} \sigma_{z}\right) i \sigma_{y}\right] \cdot \vec{\tau}
$$

While we can in general write a gap function of this form that is entirely intraband, we will for simplicity consider in the following $\delta t_{z}=0$, such that $\Delta^{\perp}(\mathbf{k})=\left(d_{\mathbf{k}}^{z} \sigma_{z}\right) i \sigma_{y} \tau_{1}$ with $d_{\mathbf{k}}^{z} \propto \sin k_{z} / 2$ as the lowest-order basis function [24]. This corresponds to an interlayer pairing between nearest layers. This gap function has a line node for $k_{z}=0$, which can be removed by mixing in the other spin-triplet component of $A_{1 u}$ symmetry, namely $\vec{d}_{\mathbf{k}}=\left(\sin k_{x} \sigma_{x}+\sin k_{y} \sigma_{y}\right) i \sigma_{y} \tau_{0}$. This order parameter thus in general allows for a full gap.

While knowledge of the pairing interaction is needed to determine the leading instability in the system, we would expect the dominant channel to be in-plane in the quasitwo-dimensional limit. Consequently, the most probable order parameters are the spin-singlet and the spin-triplet of $A_{2 u}$ symmetry. Only when approaching the three-dimensional limit, in other words $t_{z} \approx t$, does an interlayer pairing become competitive [25]. As mentioned, this last pairing channel can lead to a full gap even in the three-dimensional limit, which in turn allows for topological superconductivity, as we discuss in Sec. IV.

\section{B. General spin response}

The spin response of a superconductor can be calculated similarly to the one in the normal state, Eq. (15), using [26]

$$
\begin{aligned}
\chi_{i j}= & -\mu_{B}^{2} T \sum_{n} \sum_{\mathbf{k}} \operatorname{tr}\left[\sigma_{i} \tau_{0} G\left(\mathbf{k}, \omega_{n}\right) \sigma_{j} \tau_{0} G\left(\mathbf{k}, \omega_{n}\right)\right. \\
& \left.-\sigma_{i} \tau_{0} F\left(\mathbf{k}, \omega_{n}\right) \sigma_{j}^{T} \tau_{0} F^{\dagger}\left(\mathbf{k}, \omega_{n}\right)\right],
\end{aligned}
$$

where $G\left(\mathbf{k}, \omega_{n}\right)$ and $F\left(\mathbf{k}, \omega_{n}\right)$ are the normal and anomalous Green's functions, respectively. Given the intraband order parameters of Eqs. (20), (21), or (22), these can be calculated explicitly using the Gor'kov equations; see Appendix B. The resulting structure of both Green's functions is very similar to the normal-state Green's function of the previous section, Eq. (11). In particular, the normal Green's function reads

$$
G\left(\mathbf{k}, \omega_{n}\right)=G_{+} \sigma_{0} \tau_{0}+G_{-}\left[\hat{\xi}_{\mathbf{k}}^{-} \sigma_{0} \tau_{1}+\hat{f_{\mathbf{k}}} \cdot \vec{\sigma} \tau_{3}\right]
$$

with

$$
G_{ \pm}=G_{ \pm}\left(\mathbf{k}, \omega_{n}\right)=-\frac{1}{2}\left(\frac{i \omega_{n}+\xi_{+}}{\omega_{n}^{2}+E_{+}^{2}} \pm \frac{i \omega_{n}+\xi_{-}}{\omega_{n}^{2}+E_{-}^{2}}\right)
$$

and $E_{\alpha}=\sqrt{\xi_{\alpha}^{2}+|\Delta|^{2}}$. The normal Green's function does not depend on the specifics of the intraband gap function, other than its (momentum-dependent) magnitude, in other words $|\Delta|^{2}=|\psi|^{2}$ for the spin-singlet, $|\Delta|^{2} \equiv\left|\Delta_{\mathbf{k}}^{\|}\right|^{2}$ for the intralayer spin-triplet, and $|\Delta|^{2} \equiv\left|\Delta_{\mathbf{k}}^{\perp}\right|^{2}$ for the interlayer spin-triplet case.

For the anomalous Green's function, on the other hand, the gap structure enters explicitly,

$$
F\left(\mathbf{k}, \omega_{n}\right)=\left\{F_{+} \tau_{0}+F_{-}\left[\hat{\xi}_{\mathbf{k}}^{-} \sigma_{0} \tau_{1}+\hat{f}_{\mathbf{k}} \cdot \vec{\sigma} \tau_{3}\right]\right\} \Delta(\mathbf{k})
$$

with

$$
F_{ \pm}=F_{ \pm}\left(\mathbf{k}, \omega_{n}\right)=\frac{1}{2}\left(\frac{1}{\omega_{n}^{2}+E_{+}^{2}} \pm \frac{1}{\omega_{n}^{2}+E_{-}^{2}}\right) .
$$

To proceed, we separate the susceptibility in Eq. (23) into a "normal" and an "anomalous" part, $\chi_{i j}=\chi_{i j}^{\mathrm{n}}+\chi_{i j}^{\mathrm{a}}$. The trace of the normal part is the same for all three order parameters and, as in the discussion in the previous section, yields

$$
\frac{\chi_{z}^{\mathrm{n}}\left(\mathbf{k}, \omega_{n}\right)}{4 \mu_{B}^{2} T}=-\left\{\left(G_{+}\right)^{2}+\left(G_{-}\right)^{2}\left[\left(\hat{\xi}_{\mathbf{k}}^{-}\right)^{2}-\left|\hat{f}_{\mathbf{k}}\right|^{2}\right]\right\}
$$

and similarly

$$
\frac{\chi_{x}^{\mathrm{n}}\left(\mathbf{k}, \omega_{n}\right)}{4 \mu_{B}^{2} T}=-\left\{\left(G_{+}\right)^{2}+\left(G_{-}\right)^{2}\left[\left(\hat{\xi}_{\mathbf{k}}^{-}\right)^{2}+\left(\hat{f}_{\mathbf{k}}^{x}\right)^{2}-\left(\hat{f}_{\mathbf{k}}^{y}\right)^{2}\right]\right\} .
$$

As the gap structure enters the anomalous Green's function, we discuss in the following first the susceptibility for the case of the spin-singlet and then the case of the spin-triplet order parameters.

\section{Spin-singlet order parameter}

Using the $s$-wave singlet gap function in the anomalous Green's functions, Eq. (26), we find for the anomalous part of the susceptibility

$$
\frac{\chi_{z}^{\mathrm{a}}\left(\mathbf{k}, \omega_{n}\right)}{4 \mu_{B}^{2} T}=-|\Delta|^{2}\left\{\left(F_{+}\right)^{2}+\left(F_{-}\right)^{2}\left[\left(\hat{\xi}_{\mathbf{k}}^{-}\right)^{2}-\left|\hat{f}_{\mathbf{k}}\right|^{2}\right]\right\}
$$


and similarly

$$
\frac{\chi_{x}^{\mathrm{a}}\left(\mathbf{k}, \omega_{n}\right)}{4 \mu_{B}^{2} T}=-|\Delta|^{2}\left\{\left(F_{+}\right)^{2}+\left(F_{-}\right)^{2}\left[\left(\hat{\xi}_{\mathbf{k}}^{-}\right)^{2}+\left(\hat{f}_{\mathbf{k}}^{x}\right)^{2}-\left(\hat{f}_{\mathbf{k}}^{y}\right)^{2}\right]\right\} .
$$

We can again separate the susceptibility into two contributions, namely

$$
\begin{aligned}
\chi_{\mathrm{P}}^{+}\left(\mathbf{k}, \omega_{n}\right) & =\left(G_{+}\right)^{2}+\left(G_{-}\right)^{2}+|\Delta|^{2}\left[\left(F_{+}\right)^{2}+\left(F_{-}\right)^{2}\right] \\
& =\frac{1}{2} \sum_{\alpha= \pm} \frac{\left(i \omega_{n}+\xi_{\alpha}\right)^{2}+|\Delta|^{2}}{\left(\omega_{n}^{2}+E_{\alpha}^{2}\right)^{2}}
\end{aligned}
$$

and

$$
\begin{aligned}
\chi_{\mathrm{vV}}^{+}\left(\mathbf{k}, \omega_{n}\right) & =\left(G_{+}\right)^{2}-\left(G_{-}\right)^{2}+|\Delta|^{2}\left[\left(F_{+}\right)^{2}-\left(F_{-}\right)^{2}\right] \\
& =\frac{1}{2}\left\{\frac{\left(i \omega_{n}-\xi_{+}\right)\left(i \omega_{n}-\xi_{-}\right)+|\Delta|^{2}}{\left(\omega_{n}^{2}+E_{+}^{2}\right)\left(\omega_{n}^{2}+E_{-}^{2}\right)}\right\} .
\end{aligned}
$$

The total susceptibility after Matsubara summation is given by

$$
\chi_{z}^{\mathrm{s}}=\sum_{\mathbf{k}}\left(\hat{\xi}_{\mathbf{k}}^{-}\right)^{2} \chi_{\mathrm{P}}^{+}(\mathbf{k})+\left|\hat{f}_{\mathbf{k}}\right|^{2} \chi_{\mathrm{vV}}^{+}(\mathbf{k})
$$

for fields along $z$, and for fields along the $x$ or $y$ direction, the susceptibility has the form

$$
\chi_{x}^{\mathrm{s}}=\sum_{\mathbf{k}}\left[\left(\hat{\xi}_{\mathbf{k}}^{-}\right)^{2}+\left(\hat{f}_{\mathbf{k}}^{x}\right)^{2}\right] \chi_{\mathrm{P}}^{+}(\mathbf{k})+\left(\hat{f}_{\mathbf{k}}^{y}\right)^{2} \chi_{\mathrm{vV}}^{+}(\mathbf{k})
$$

The Matsubara sums are evaluated in Appendix $\mathrm{C}$ and yield

$$
\chi_{\mathrm{P}}^{+}(\mathbf{k})=2 \mu_{B}^{2} \sum_{\alpha} \frac{1}{4 T \cosh ^{2}\left(E_{\alpha} / 2 T\right)}
$$

for the Pauli term, and for $\Delta \ll \sqrt{\left|\vec{\xi}_{\mathbf{k}}^{-}\right|^{2}+\left|\vec{f}_{\mathbf{k}}\right|^{2}}$ we can approximate

$$
\chi_{\mathrm{vV}}^{+}(\mathbf{k}) \approx 2 \mu_{B}^{2} \frac{\left[n_{\mathrm{F}}\left(E_{+}\right)-n_{\mathrm{F}}\left(E_{-}\right)\right]}{\sqrt{\left|\vec{f}_{\mathbf{k}}\right|^{2}+\left|\vec{\xi}_{\mathbf{k}}^{-}\right|^{2}}} \approx \chi_{\mathrm{vV}}^{0}(\mathbf{k})
$$

for the van Vleck susceptibility.

\section{Intralayer spin-triplet pairing}

For spin-triplet pairing, we start with the intralayer-pairing case with order parameter $\Delta^{\|}(\mathbf{k})=\left(\vec{d}_{\mathbf{k}}^{\|} \cdot \vec{\sigma}\right) i \sigma_{y} \tau_{0}$, where $\vec{d}_{\mathbf{k}}^{\|} \|$

$$
\left.\chi_{x}^{\mathrm{t}, \|}=\sum_{\mathbf{k}}\left\{\left[\left(\hat{\xi}_{\mathbf{k}}^{-}\right)^{2}+\left(\hat{f}_{\mathbf{k}}^{x}\right)^{2}\right] \frac{\left|d_{\mathbf{k}}^{x}\right|^{2}}{\left|\Delta_{\mathbf{k}}\right|^{2}} \chi_{\mathrm{P}}^{+}(\mathbf{k})+\frac{\left|d_{\mathbf{k}}^{y}\right|^{2}}{\left|\Delta_{\mathbf{k}}\right|^{2}} \chi_{\mathrm{P}}^{-}(\mathbf{k})\right]+\left(\hat{f}_{\mathbf{k}}^{y}\right)^{2} \chi_{\mathrm{vV}}(\mathbf{k})\right\} .
$$

\section{Interlayer pairing}

For the interlayer pairing with $\Delta^{\perp}(\mathbf{k})=\left(d_{\mathbf{k}}^{z} \sigma_{z}\right) i \sigma_{y} \tau_{1}$, the traces of the anomalous part of the susceptibility in Eq. (23) yield for out-of-plane fields

$$
\frac{\chi_{z}^{\mathrm{a}}}{4 \mu_{B}^{2} T}=-\left|d_{\mathbf{k}}^{z}\right|^{2}\left\{F_{+}^{2}+F_{-}^{2}\left[\left(\hat{\xi}_{\mathbf{k}}^{-}\right)^{2}-\left|\hat{f}_{\mathbf{k}}\right|^{2}\right]\right\}
$$

$\vec{f}_{\mathbf{k}}$. For out-of-plane fields, $i=j=z$, the anomalous part of the susceptibility yields

$$
\frac{\chi_{z}^{\mathrm{a}}}{4 \mu_{B}^{2} T}=\left|\Delta_{\mathbf{k}}\right|^{2}\left\{F_{+}^{2}+F_{-}^{2}\left[\left(\hat{\xi}_{\mathbf{k}}^{-}\right)^{2}-\left|\hat{f}_{\mathbf{k}}\right|^{2}\right]\right\} .
$$

For in-plane fields, $i=j=x$, we find

$$
\frac{\chi_{x}^{\mathrm{a}}}{4 \mu_{B}^{2} T}=\left(\left|d_{\mathbf{k}}^{y}\right|^{2}-\left|d_{\mathbf{k}}^{x}\right|^{2}\right)\left\{F_{+}^{2}+F_{-}^{2}\left[\left(\hat{\xi}_{\mathbf{k}}^{-}\right)^{2}+\left(\hat{f}_{\mathbf{k}}^{x}\right)^{2}-\left(\hat{f}_{\mathbf{k}}^{y}\right)^{2}\right]\right\} \text {. }
$$

Combining Eqs. (28) and (29) with (38) and (39), we thus find for the total susceptibility two additional contributions compared to the spin-singlet case. First,

$$
\begin{aligned}
\chi_{\mathrm{P}}^{-}\left(\mathbf{k}, \omega_{n}\right) & =\left(G_{+}\right)^{2}+\left(G_{-}\right)^{2}-|\Delta|^{2}\left[\left(F_{+}\right)^{2}+\left(F_{-}\right)^{2}\right] \\
& =\frac{1}{2} \sum_{\alpha= \pm} \frac{\left(i \omega_{n}+\xi_{\alpha}\right)^{2}-|\Delta|^{2}}{\left(\omega_{n}^{2}+E_{\alpha}^{2}\right)^{2}}
\end{aligned}
$$

which, after performing the Matsubara sum (Appendix C), yields

$$
\chi_{\mathrm{P}}^{-}(\mathbf{k})=2 \mu_{B}^{2} \sum_{\alpha} \frac{|\Delta|^{2}}{E_{\alpha}^{3}} \tanh \left(\frac{E_{\alpha}}{2 T}\right)+\frac{\xi_{\alpha}^{2}}{E_{\alpha}^{2}} \frac{1}{4 T \cosh ^{2}\left(E_{\alpha} / 2 T\right)} .
$$

The second,

$$
\begin{aligned}
\chi_{\mathrm{vV}}^{-}\left(\mathbf{k}, \omega_{n}\right) & =\left(G_{+}\right)^{2}-\left(G_{-}\right)^{2}-|\Delta|^{2}\left[\left(F_{+}\right)^{2}-\left(F_{-}\right)^{2}\right] \\
& =\frac{1}{2}\left\{\frac{\left(i \omega_{n}-\xi_{+}\right)\left(i \omega_{n}-\xi_{-}\right)-|\Delta|^{2}}{\left(\omega_{n}^{2}+E_{+}^{2}\right)\left(\omega_{n}^{2}+E_{-}^{2}\right)}\right\}
\end{aligned}
$$

yields for $\sqrt{\left|\vec{\xi}_{\mathbf{k}}^{-}\right|^{2}+\left|\vec{f}_{\mathbf{k}}\right|^{2}} \gg \Delta$ the same van Vleck contribution as Eq. (37), $\chi_{\mathrm{vV}}^{-}(\mathbf{k}) \approx \chi_{\mathrm{vV}}^{+}(\mathbf{k}) \equiv \chi_{\mathrm{vV}}(\mathbf{k})$. For fields out of plane, we thus find the susceptibility

$$
\chi_{z}^{\mathrm{t}, \|}=\sum_{\mathbf{k}}\left(\hat{\xi}_{\mathbf{k}}^{-}\right)^{2} \chi_{\mathrm{P}}^{-}(\mathbf{k})+\left|\hat{f}_{\mathbf{k}}\right|^{2} \chi_{\mathrm{vV}}(\mathbf{k})
$$

and for the susceptibility for in-plane fields and for in-plane fields, $i=j=x$, we find

$$
\frac{\chi_{x}^{\mathrm{a}}}{4 \mu_{B}^{2} T}=\left|d_{\mathbf{k}}^{z}\right|^{2}\left\{F_{+}^{2}+F_{-}^{2}\left[\left(\hat{\xi}_{\mathbf{k}}^{-}\right)^{2}+\left(\hat{f}_{\mathbf{k}}^{x}\right)^{2}-\left(\hat{f}_{\mathbf{k}}^{y}\right)^{2}\right]\right\} .
$$

For fields out-of-plane, we thus find the susceptibility

$$
\chi_{z}^{\mathrm{t}, \perp}=\sum_{\mathbf{k}}\left(\hat{\xi}_{\mathbf{k}}^{-}\right)^{2} \chi_{\mathrm{P}}^{+}(\mathbf{k})+\left|\hat{f}_{\mathbf{k}}\right|^{2} \chi_{\mathrm{vV}}(\mathbf{k})
$$




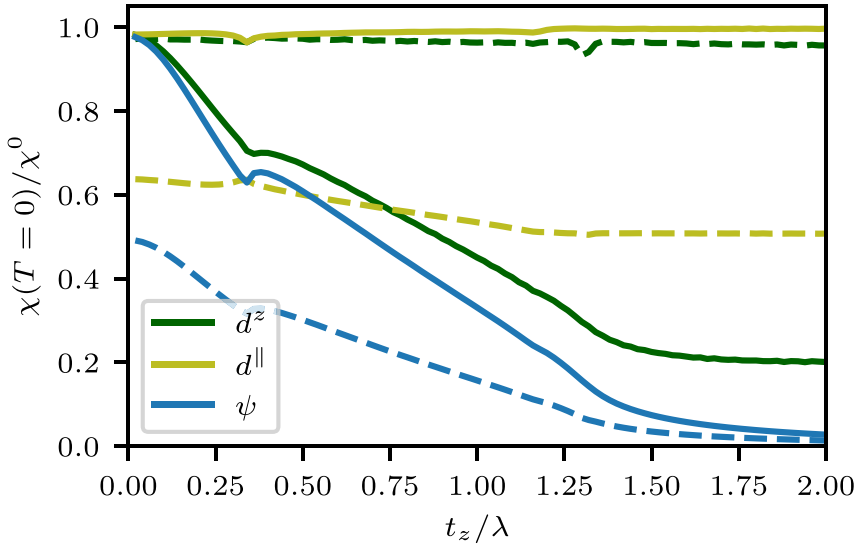

FIG. 3. Out-of-plane (solid lines) and in-plane (dashed lines) susceptibility for all three order parameters for $T \rightarrow 0$ compared to the normal-state susceptibility as a function of $z$ axis hopping for $\delta t_{z}=0$. The pairing gaps are defined as $\Delta(\mathbf{k})=\psi$ for the spin-singlet, $\vec{d}_{\mathbf{k}}^{\|}=\Delta\left(-\sin k_{y}, \sin k_{x}, 0\right)$ for intralayer pairing, and $d_{\mathbf{k}}^{z}=\Delta \sin k_{z} / 2$ for interlayer pairing. For the numerical evaluation, we used $\Delta=0.025 t$ and $T=0.0025 t$.

and for in-plane fields the susceptibility

$$
\chi_{x}^{\mathrm{t}, \perp}=\sum_{\mathbf{k}}\left\{\left[\left(\hat{\xi}_{\mathbf{k}}^{-}\right)^{2}+\left(\hat{f}_{\mathbf{k}}^{x}\right)^{2}\right] \chi_{\mathrm{P}}^{-}(\mathbf{k})+\left(\hat{f}_{\mathbf{k}}^{y}\right)^{2} \chi_{\mathrm{vV}}(\mathbf{k})\right\} .
$$

\section{Discussion}

Because the Pauli susceptibility in Eq. (36) vanishes for $T \rightarrow 0$ for the spin-singlet state, only the temperatureindependent van Vleck contribution of the normal state remains. Moreover, comparing Eqs. (34) and (35), we find for the spin-singlet pairing $\chi_{z}^{\mathrm{s}}=2 \chi_{x}^{\mathrm{s}}$. To discuss the behavior of the susceptibility for the triplet states in the $T \rightarrow 0$ limit, we rewrite the term in Eq. (41) as

$$
\chi_{\mathrm{P}}^{-}(\mathbf{k})=2 \mu_{B}^{2} \sum_{\alpha} \frac{\partial}{\partial \xi}\left[\frac{\xi_{\alpha}}{E_{\alpha}} \tanh \left(\frac{E_{\alpha}}{2 T}\right)\right] \approx \chi_{\mathrm{P}}^{0}(\mathbf{k}),
$$

which, for $T \ll \Delta \ll t$, reduces to a derivative of a step function around $\mu$ and thus approximately to the normal-state Pauli contribution to the susceptibility. Hence, we find that the spin susceptibility for out-of-plane fields does not decrease for intralayer pairing, while it follows the trend of the spin-singlet case for interlayer pairing. For in-plane fields, however, the intralayer pairing state reduces the spin susceptibility, while the interlayer pairing state is not paramagnetically limited. These findings are thus in accordance with the general expectation that for a $d$-vector perpendicular to the magnetic field, the susceptibility stays constant, while the contribution parallel to the field reduces the susceptibility. Note that here, the van Vleck term is generally not affected by superconductivity, since it arises from interband contributions, and the band splitting is governed by $\sqrt{\left|\vec{\xi}_{\mathbf{k}}^{-}\right|^{2}+\left|\vec{f}_{\mathbf{k}}\right|^{2}} \gg \Delta$.

Figure 3 shows the susceptibilities for all three order parameters as a function of the $z$-axis hopping $t_{z}$, where, as mentioned, we use $\delta t_{z}=0$ for simplicity. As discussed above, the zero-temperature in-plane susceptibility is always reduced to half of the out-of-plane susceptibility for (fully gapped) spin-singlet pairing, while this is not the case for spin-triplet order parameters. Note that the interlayer state has a larger susceptibility in these calculations due to the line nodes at $k_{z}=0$. These nodes can in principle be gapped out, such that the out-of-plane susceptibility of the spin-singlet and the interlayer spin-triplet would be equal. Finally, note that the in-plane (out-of-plane) pairing state is limited by orbital depairing for out-of-plane (in-plane) fields.

\section{TOPOLOGICAL CONSIDERATIONS}

In this section, we investigate the possible nontrivial topology of the superconducting phases in the different regimes identified in Sec. II. In systems invariant under inversion with an odd-parity superconducting order parameter, the topology of the superconducting phase can be identified using so-called symmetry indicators (SI) [15-17]. These indicators are defined in terms of the inversion eigenvalues of the occupied normal-state bands at time-reversal-symmetric momenta (TRIMs), where $-\mathbf{k} \equiv \mathbf{k}$ up to reciprocal-lattice translations. In particular, the $3 \mathrm{D}$ inversion-symmetry indicator $\kappa_{3 \mathrm{D}}$ is a $\mathbb{Z}_{8}$-valued quantity, such that an odd SI indicates a strong topological superconductor, while $\kappa_{3 \mathrm{D}}= \pm 2$ and $\kappa_{3 \mathrm{D}}=4$ indicate second- and third-order topological phases, respectively.

The inversion-symmetry indicator for a time-reversal symmetric odd-parity superconductor is defined as

$$
\kappa_{3 D}=\frac{1}{2} \sum_{\mathbf{k} \in \mathrm{TRIMs}}\left(n_{\mathbf{k}, \mathrm{N}}^{+}-n_{\mathbf{k}, \mathrm{N}}^{-}\right),
$$

where $n_{\mathbf{k}, \mathrm{N}}^{ \pm}$is the number of occupied bands at the TRIMs with inversion eigenvalue \pm 1 . To calculate the indicator, it is convenient to rewrite the dispersion in the $z$ direction, Eqs. (7) and (8), as

$$
\begin{gathered}
\left(\tilde{\xi}_{\mathbf{k}}^{-}\right)_{1}=-t_{z}+\delta t_{z}-\left(t_{z}+\delta t_{z}\right) \cos k_{z}, \\
\left(\tilde{\xi}_{\mathbf{k}}^{-}\right)_{2}=-\left(t_{z}+\delta t_{z}\right) \sin k_{z} .
\end{gathered}
$$

This corresponds to a gauge transformation such that the Fourier transform does not resolve the unit-cell structure anymore. The advantage, however, is that now $\mathcal{I H}_{\mathbf{k}} \mathcal{I}^{-1}=\mathcal{H}_{-\mathbf{k}}$ with $\mathcal{I}=\sigma_{0} \tau_{1}$ and we can straightforwardly read off the inversion eigenvalues of the normal-state bands.

We are interested in inversion eigenvalues at two TRIMs $\Gamma=(0,0,0)$ and $Z=(0,0, \pi)$. At these two momenta, the two bands with energies given by Eq. (9), namely $\xi_{\Gamma, \pm}=$ $-4 t \mp 2 t_{z}$ and $\xi_{Z, \pm}=-4 t \mp 2 \delta t_{z}$, have inversion eigenvalues \pm 1 . Depending on the Fermi-surface topology and hence the number of bands occupied at the TRIMs, the system can realize a first- or a second-order topological superconductor; see Fig. 4. A third-order topological superconductor, however, is not possible at least with this simplified band structure, since for both bands occupied, the inversion eigenvalues are opposite and cancel in Eq. (50).

Table I summarizes the symmetry indicators for the two spin-triplet (odd-parity) order parameters considered here. In particular, the $A_{2 u}$ state, which pairs electrons within the layers and is thus more probable in the q2D limit, allows for a second-order phase when only one open Fermi surface exists. 

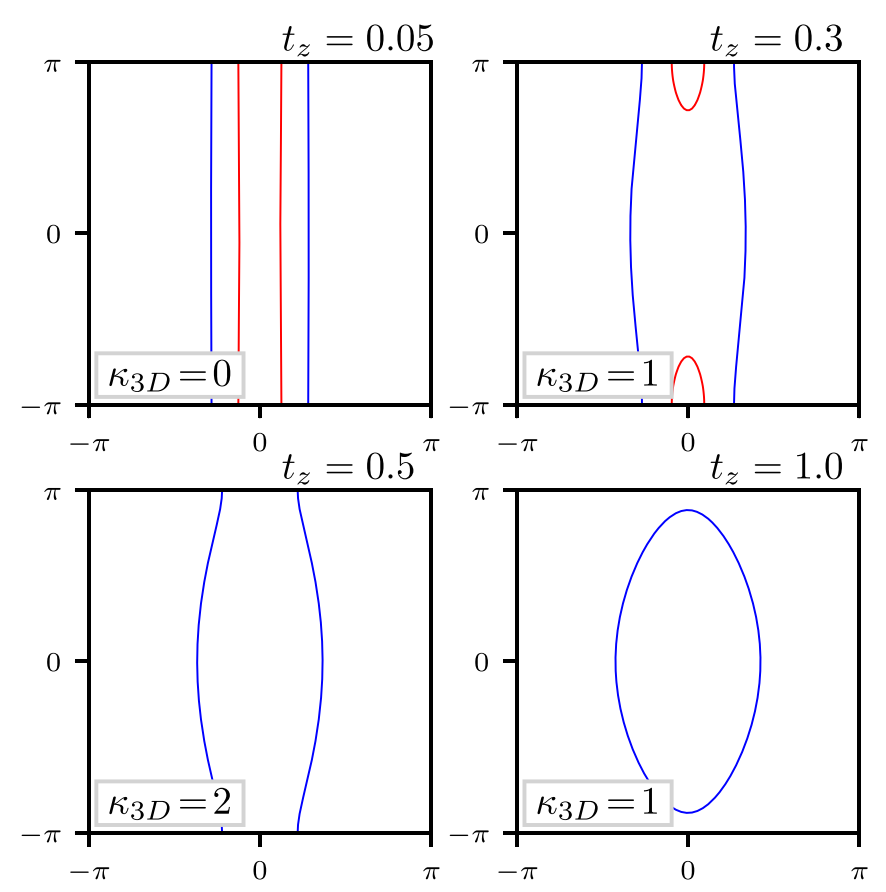

FIG. 4. Projection of the Fermi surface onto the $k_{x}-k_{z}$ plane for different values of interlayer hopping parameters $t_{z}$ and $\delta t_{z}$. Two colors correspond to two different momentum sectors with inversion eigenvalues +1 and -1 , respectively.

In the other potentially nontrivial cases, this gap structure has point nodes for $k_{x}=k_{y}=0$. The $A_{1 u}$ state, which can be fully gapped for any Fermi-surface topology, thus allows in principle for both first- and second-order phases. As the $A_{1 u}$ state corresponds to an anisotropic Balian-Werthammer state known from the $\mathrm{B}$ phase of ${ }^{3} \mathrm{He}$, a nontrivial topology might not be surprising [27]. Note, however, that this gap structure is rather unlikely in the quasi-2D limit.

\section{CONCLUSION}

In some globally centrosymmetric systems, the lack of inversion symmetry in subunits can influence the physical properties significantly. Such remnants of noncentrosymmetricity are particular interesting in the context of superconductivity, where inversion symmetry yields a strict distinction between spin-singlet and spin-triplet Cooper pairing. In locally noncentrosymmetric systems, however, the two may mix in a very characteristic way.

TABLE I. Symmetry indicator for the two spin-triplet order parameters with $A_{1 u}$ and $A_{2 u}$ symmetry, where 1 corresponds to a first-order and 2 to a second-order topological superconductor. The $\times$ indicates that the gap has point nodes and no strong topology is possible, while parentheses indicates that the interlayer order parameter is not likely unless the system is three-dimensional.

\begin{tabular}{ccccc}
\hline \hline$\Delta$ & q2D & q3DI & q3DII & $3 \mathrm{D}$ \\
\hline$A_{1 u}$ & $(0)$ & $(1)$ & 2 & 1 \\
$A_{2 u}$ & 0 & $\times$ & 2 & $\times$ \\
\hline \hline
\end{tabular}

In our work, we have investigated signatures of local noncentrosymmetricity for the case of a layered system with staggered layer-intrinsic Rashba spin-orbit coupling. Hereby, we have focused on different regimes going from a quasi$2 \mathrm{D}$ to fully $3 \mathrm{D}$ band structure upon tuning the interlayer hybridization. First looking at the normal-state spin susceptibility, we identified four different regimes, characterized by their Fermi surface topology.

Unlike the case of globally noncentrosymmetric superconductors, where only one kind of spin-triplet state is feasible, we identified two such spin-triplet states in the staggered case. We analyzed the resulting three order parameters, namely a generic spin-singlet and those two spin-triplet phases, and we show that the comparison between their behavior under in-plane and out-of-plane magnetic fields allows for the order parameters' distinction. In particular, their low-temperature spin susceptibility displays different behavior that influence the paramagnetic limiting effects for different field orientations.

For the spin-triplet order parameters, we have finally explored possible topological phases within the symmetry indicator framework, and we identified both first- and secondorder topological phases. Third-order topology is, on the other hand, not possible in the minimal model considered here.

Finally, we comment on the relevance of our results concerning $\mathrm{CeRh}_{2} \mathrm{As}_{2}$ [12]. While our study cannot quantitatively account for either the complicated band structure or interaction effects in this heavy-fermion compound, qualitative conclusions can be drawn [28]: For $c$-axis fields, the low-field state seems to be paramagnetically limited, which could have either spin-singlet or interlayer spin-triplet character. However, as the in-plane fields show a smaller critical field for $\mathrm{CeRh}_{2} \mathrm{As}_{2}$, it is rather likely that the low-field phase constitutes spin-singlet pairing. Note that the spin-triplet phase that would emerge in this scenario at high fields is certainly topologically trivial, as there are no first-order TSC in 3D. A generic possibility would, however, be a Weyl superconductor. As the field-induced phase transition indicates that the spintriplet state is close by in parameter space, it might be possible to stabilize it also with other means, thus inducing possibly topological superconductivity.

\section{ACKNOWLEDGMENTS}

We thank Daniel F. Agterberg, Elena Hassinger, Seunghyun Khim, Titus Neupert, Eric Schertenleib, and Luka Trifunovic for fruitful discussions. A.S. was supported by funding from the European Research Council (ERC) under the European Unions Horizon 2020 research and innovation program (ERC-StG-Neupert-757867-PARATOP) and by a Forschungskredit of the University of Zurich, Grant No. FK-20-101. M.S. is financially supported by a Grant of the Swiss National Science Foundation (No. 184739).

\section{APPENDIX A: NORMAL-STATE SUSCEPTIBILITY}

As shown in the main text, there are two contributions to the normal-state susceptibility $\chi^{0}(\mathbf{k})$ : The Pauli $\chi_{\mathrm{P}}^{0}(\mathbf{k})$ and the van Vleck $\chi_{\mathrm{vV}}^{0}(\mathbf{k})$ contribution. The two contributions behave 


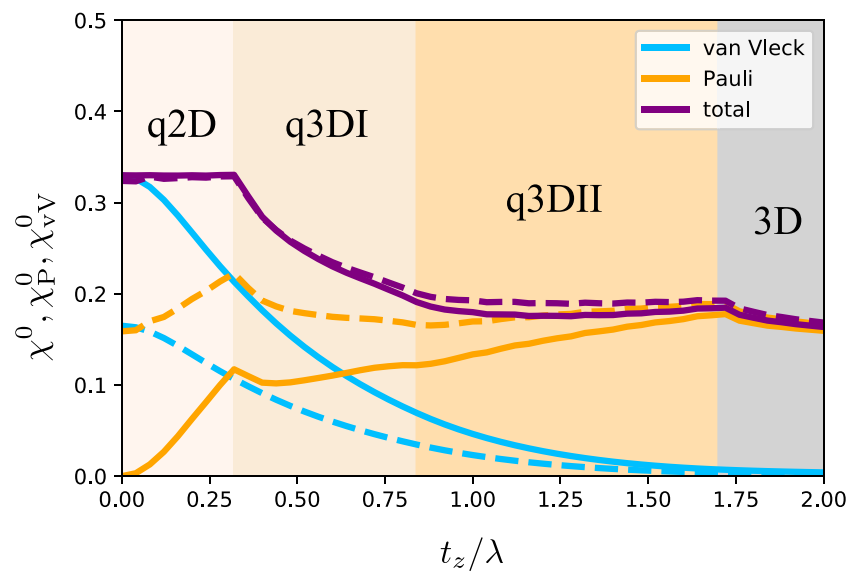

FIG. 5. The normal state susceptibility, and the Pauli and the van Vleck contributions to it for fields along the $z$ direction (solid lines) and in-plane (dashed lines). Four different regimes of the Fermi surface topology are indicated for the case of $\delta t_{z} / t_{z}=0.2$. For numerical reasons, we set $T=0.0025 t$.

differently as the system goes from quasi-2D to fully bulk-3D behavior.

At low interlayer coupling $t_{z}$, normal-state susceptibility is fully dominated by the van Vleck term for in-plane fields, while in the case of out-of-plane fields it is composed by equal contributions from both the Pauli and the van Vleck terms. As the interlayer coupling $t_{z}$ increases, the van Vleck contribution vanishes while the Pauli term increases. The exact behavior of the Pauli term, in particular its cusps, is strongly affected by the Lifshitz transitions resulting in the change of the Fermi surface topology (see Fig. 5).

In the main text, we used an electron density fixed at $n_{\text {tot }}=0.15$ per unit cell. While the qualitative picture does not change when increasing the electron density, larger inter-plane hopping $t_{z}$ is required to go through the Lifshitz transitions and access a more three-dimensional character of the system. This is illustrated in Fig. 6, where the first peak in the Pauli susceptibility shifts to higher $t_{z} / \lambda$ for increased density. Note that the peak at $t_{z} / \lambda \approx 1.75$ for $n_{\text {tot }}=0.15$ is connected to entering the truly $3 \mathrm{D}$ regime (see also Fig. 2) and can only be accessed in the parameter regime studied for this low electron density.

\section{APPENDIX B: GOR'KOV EQUATIONS}

To calculate the Green's functions, we can use the Gor'kov equations

$$
\begin{gathered}
G_{0}^{-1}\left(\mathbf{k}, \omega_{n}\right) G\left(\mathbf{k}, \omega_{n}\right)+\Delta_{\mathbf{k}} F^{\dagger}\left(\mathbf{k}, \omega_{n}\right)=\tau_{0} \sigma_{0}, \\
\left(G_{0}^{-1}\right)^{T}\left(-\mathbf{k},-\omega_{n}\right) F^{\dagger}\left(\mathbf{k}, \omega_{n}\right)-\Delta_{\mathbf{k}}^{\dagger} G\left(\mathbf{k}, \omega_{n}\right)=0, \\
G_{0}^{-1}\left(\mathbf{k}, \omega_{n}\right) F\left(\mathbf{k}, \omega_{n}\right)-\Delta_{\mathbf{k}} G^{T}\left(-\mathbf{k},-\omega_{n}\right)=0 .
\end{gathered}
$$

Equation (B2) leads to

$$
F^{\dagger}\left(\mathbf{k}, \omega_{n}\right)=G_{0}^{T}\left(-\mathbf{k},-\omega_{n}\right) \Delta_{\mathbf{k}}^{\dagger} G\left(\mathbf{k}, \omega_{n}\right) .
$$

We can use this in the first Gor'kov equation to find

$$
G^{-1}\left(\mathbf{k}, \omega_{n}\right)=G_{0}^{-1}\left(\mathbf{k}, \omega_{n}\right)+\Delta_{\mathbf{k}} G_{0}^{T}\left(-\mathbf{k},-\omega_{n}\right) \Delta_{\mathbf{k}}^{\dagger} .
$$

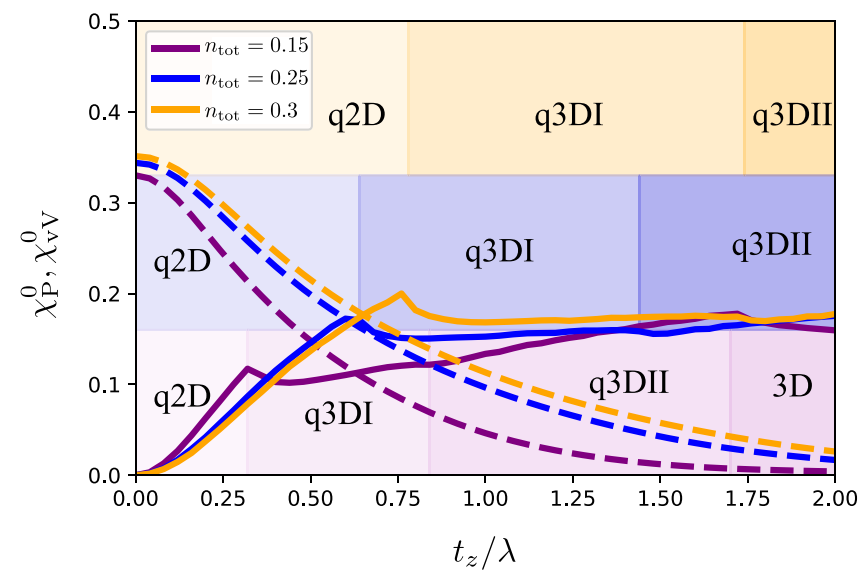

FIG. 6. The Pauli (solid lines) and the van Vleck (dashed lines) contributions to the normal state susceptibility for fields along the $z$ direction for $\delta t_{z} / t_{z}=0.2$. Colored regions indicate regimes with different Fermi surface topology for three values of the electron density $n_{\text {tot }}$.

For the following, we thus need the normal-state Green's function given by Eq. (11) and similarly

$$
G_{0}^{T}\left(-\mathbf{k},-\omega_{n}\right)=\tilde{G}_{+}^{0} \sigma_{0} \tau_{0}+\tilde{G}_{-}^{0}\left[\hat{\xi}_{\mathbf{k}}^{-} \sigma_{0} \tau_{3}+\left(\hat{f}_{\mathbf{k}}^{y} \sigma_{y}-\hat{f}_{\mathbf{k}}^{x} \sigma_{x}\right) \tau_{1}\right]
$$

$\left[\tilde{G}_{ \pm}^{0}=G_{ \pm}^{0}\left(-\mathbf{k},-\omega_{n}\right)\right.$ and $\left.\vec{f}_{-\mathbf{k}}=-\vec{f}_{\mathbf{k}}\right]$. We are interested in the intraband gap functions given in Eqs. (20) or (21) and (22), for which we find

$$
\begin{aligned}
& \Delta_{\mathbf{k}} G_{0}^{T}\left(-\mathbf{k},-\omega_{n}\right) \Delta_{\mathbf{k}}^{\dagger} \\
& \quad=\left|\Delta_{\mathbf{k}}\right|^{2}\left[\tilde{G}_{+}^{0} \sigma_{0} \tau_{0}+\tilde{G}_{-}^{0}\left[\hat{\xi}_{\mathbf{k}}^{-} \sigma_{0} \tau_{3}+\hat{\overrightarrow{f_{\mathbf{k}}}} \cdot \vec{\sigma} \tau_{1}\right]\right]
\end{aligned}
$$

with $\Delta_{\mathbf{k}} \Delta_{\mathbf{k}}^{\dagger}=\left|\Delta_{\mathbf{k}}\right|^{2} \sigma_{0} \tau_{0}$. Then

$$
\begin{aligned}
G^{-1}\left(\mathbf{k}, \omega_{n}\right)= & \left(i \omega_{n}-\xi_{\mathbf{k}}^{+}+\Delta^{2} \tilde{G}_{+}^{0}\right) \tau_{0} \sigma_{0}+\left(\left|\Delta_{\mathbf{k}}\right|^{2} \tilde{G}_{-}^{0}\right. \\
& \left.-\sqrt{\left|\vec{f}_{\mathbf{k}}\right|^{2}+\left(\xi_{\mathbf{k}}^{-}\right)^{2}}\right)\left[\hat{\xi}_{\mathbf{k}}^{-} \sigma_{0} \tau_{3}+\hat{\overrightarrow{f_{\mathbf{k}}}} \cdot \vec{\sigma} \tau_{1}\right] .
\end{aligned}
$$

To invert this expression, we note that for each $\mathbf{k}$, we can rotate the spins to be parallel to $z$ such that the inverse Green's function (B8) has the structure

$$
G^{-1}\left(\mathbf{k}, \omega_{n}\right)=A \tau_{0}+B \hat{B}_{s} \cdot \tau
$$

with $\hat{B}_{s}$ a unit vector in the space of $\tau$ matrices for spin $s$. The inversion is then given by

$$
G\left(\mathbf{k}, \omega_{n}\right)=G_{+} \tau_{0}+G_{-} \hat{B}_{s} \cdot \tau
$$

with

$$
G_{ \pm}=\frac{1}{2}\left(\frac{1}{A+B} \pm \frac{1}{A-B}\right)
$$

Finally, we find

$$
\begin{aligned}
A \pm B & =i \omega_{n}-\xi_{\mathbf{k}}^{+}+\left|\Delta_{\mathbf{k}}\right|^{2}\left(\tilde{G}_{+}^{0} \pm \tilde{G}_{-}^{0}\right) \mp \sqrt{\left|\vec{f}_{\mathbf{k}}\right|^{2}+\left(\xi_{\mathbf{k}}^{-}\right)^{2}} \\
& =-\frac{\omega_{n}^{2}+\xi_{ \pm}^{2}+\left|\Delta_{\mathbf{k}}\right|^{2}}{i \omega_{n}+\xi_{ \pm}},
\end{aligned}
$$


such that, after rotating the spin back, the normal Green's function in the superconducting phase is given by Eq. (24).
Using Eq. (B3), we can further calculate the anomalous Green's function to arrive at Eqs. (26) and (27).

\section{APPENDIX C: MATSUBARA SUMS OF SUPERCONDUCTING GREEN'S FUNCTIONS}

The susceptibilities we calculated in the main text comprise four different terms of the general form denoted by $\chi_{\mathrm{P}}^{ \pm}$and $\chi_{\mathrm{vV}}^{ \pm}$. In this Appendix, we will explicitly perform the Matsubara sums to better understand these contributions.

\section{Pauli-like terms}

To evaluate the Matsubara sum of the first two contributions, Eqs. (36) and (41), we need to calculate the residues of

$$
\frac{\left(z+\xi_{\lambda}\right)^{2} \pm\left|\Delta_{\mathbf{k}}\right|^{2}}{\left(-z^{2}+\xi_{\lambda}^{2}+\left|\Delta_{\mathbf{k}}\right|^{2}\right)^{2}} n_{\mathrm{F}}(z)
$$

at the singularities not stemming from the Fermi distribution function. We find two second-order poles, namely $z= \pm E=$ $\pm \sqrt{\xi^{2}+|\Delta|^{2}}$ (for simplicity, we omit the index $\alpha$ here). The residue is then

$$
\begin{aligned}
\operatorname{Res}_{ \pm E} & =\lim _{z \rightarrow \pm E} \frac{\partial}{\partial z}\left[\frac{(z+\xi)^{2}+|\Delta|^{2}}{(z \pm E)^{2}} n_{\mathrm{F}}(z)\right] \\
& =\lim _{z \rightarrow \pm E}\left[\frac{2 z+2 \xi}{(z \pm E)^{2}} n_{\mathrm{F}}(z)-2 \frac{(z+\xi)^{2}+|\Delta|^{2}}{(z \pm E)^{3}} n_{\mathrm{F}}(z)+\frac{(z+\xi)^{2}+|\Delta|^{2}}{(z \pm E)^{2}} n_{\mathrm{F}}^{\prime}(z)\right] \\
& =\left[\frac{ \pm 2 E+2 \xi}{4 E^{2}} n_{\mathrm{F}}( \pm E)-\frac{( \pm E+\xi)^{2}+|\Delta|^{2}}{ \pm 4 E^{3}} n_{\mathrm{F}}( \pm E)+\frac{( \pm E+\xi)^{2}+|\Delta|^{2}}{4 E^{2}} n_{\mathrm{F}}^{\prime}( \pm E)\right] \\
& =\left[ \pm \frac{2 E^{2} \pm 2 E \xi-E^{2} \mp 2 E \xi-\xi^{2}-|\Delta|^{2}}{4 E^{3}} n_{\mathrm{F}}( \pm E)+\frac{( \pm E+\xi)^{2}+|\Delta|^{2}}{4 E^{2}} n_{\mathrm{F}}^{\prime}( \pm E)\right] \\
& =\left[ \pm \frac{E^{2}-\xi^{2}-|\Delta|^{2}}{4 E^{3}} n_{\mathrm{F}}( \pm E)+\frac{( \pm E+\xi)^{2}+|\Delta|^{2}}{4 E^{2}} n_{\mathrm{F}}^{\prime}( \pm E)\right] .
\end{aligned}
$$

Using that

$$
n_{\mathrm{F}}^{\prime}(z)=\frac{\partial n_{\mathrm{F}(\mathrm{z})}}{\partial z}=\frac{1}{4 T \cosh ^{2}(z / 2 T)}
$$

is an even function of $z$, we find for the sum of the two residues

$$
\operatorname{Res}_{E}+\operatorname{Res}_{-E}=\frac{1}{4 T \cosh ^{2}(E / 2 T)} .
$$

Similarly, replacing $|\Delta|^{2}$ with $-|\Delta|^{2}$ in Eq. (C2) for $\chi_{\mathrm{P}}^{-}$, we find

$$
\operatorname{Res}_{E}+\operatorname{Res}_{-E}=\frac{|\Delta|^{2}}{E^{3}} \tanh \left(\frac{E}{2 T}\right)+\frac{\xi^{2}}{E^{2}} \frac{1}{4 T \cosh ^{2}(E / 2 T)} .
$$

Finally, we write for the two Pauli contributions

$$
\begin{gathered}
\chi_{\mathrm{P}}^{+}(\mathbf{k})=2 \mu_{B}^{2} \sum_{\lambda} \frac{1}{4 T \cosh ^{2}\left(E_{\lambda} / 2 T\right)}, \\
\chi_{\mathrm{P}}^{-}(\mathbf{k})=2 \mu_{B}^{2} \sum_{\lambda} \frac{|\Delta|^{2}}{E_{\lambda}^{3}} \tanh \left(\frac{E_{\lambda}}{2 T}\right)+\frac{\xi_{\lambda}^{2}}{E_{\lambda}^{2}} \frac{1}{4 T \cosh ^{2}\left(E_{\lambda} / 2 T\right)} .
\end{gathered}
$$

The terms with the cosh vanish for $T \rightarrow 0$, such that only the first term of the latter equation survives.

\section{2. van Vleck-like terms}

The second set of contributions, Eqs. (37) and (42), has four poles at $\pm E_{ \pm}$and they are all first order. At $T=0$, the poles with positive energy thus lead to a vanishing Fermi function while the Fermi functions for the negative energies yield 1. We thus find

$$
\chi_{\mathrm{vV}}^{ \pm}(\mathbf{k})=2 \mu_{B}^{2} \sum_{\alpha= \pm} \alpha \frac{\left(E_{\alpha}+\xi_{+}\right)\left(E_{\alpha}+\xi_{-}\right) \pm|\Delta|^{2}}{2 E_{\alpha}\left(E_{+}-E_{-}\right)\left(E_{+}+E_{-}\right)}
$$


The energy scale for the van Vleck susceptibility is given by $\sqrt{\left|\vec{f}_{\mathbf{k}}\right|^{2}+\left|\vec{\xi}_{\mathbf{k}}^{-}\right|^{2}}$, and since we assume $\sqrt{\left|\vec{f}_{\mathbf{k}}\right|^{2}+\left|\vec{\xi}_{\mathbf{k}}^{-}\right|^{2}} \gg \Delta$, we proceed by setting the gap to zero. Then, we find

$$
\chi_{\mathrm{vV}}^{ \pm}(\mathbf{k}) \approx \chi_{\mathrm{vV}}(\mathbf{k})=2 \mu_{B}^{2}\left[\frac{\left(\left|\xi_{+}\right|+\xi_{+}\right)\left(\left|\xi_{+}\right|+\xi_{-}\right)}{2\left|\xi_{+}\right| \xi_{\mathbf{k}}^{+} \sqrt{\left|\vec{f}_{\mathbf{k}}\right|^{2}+\left|\vec{\xi}_{\mathbf{k}}^{-}\right|^{2}}}-\frac{\left(\left|\xi_{-}\right|+\xi_{+}\right)\left(\left|\xi_{-}\right|+\xi_{-}\right)}{2\left|\xi_{-}\right| \xi_{\mathbf{k}}^{+} \sqrt{\left|\vec{f}_{\mathbf{k}}\right|^{2}+\left|\vec{\xi}_{\mathbf{k}}^{-}\right|^{2}}}\right] .
$$

This expression yields exactly zero if both $\xi_{+}$and $\xi_{-}$are either positive or negative. However, when only one of them is negative while the other is positive, i.e., $n_{F}\left(\xi_{+}\right)-n_{F}\left(\xi_{-}\right) \neq 0$, we find

$$
\chi_{\mathrm{vV}}(\mathbf{k}) \approx 2 \mu_{B}^{2} \frac{\left[n_{F}\left(\xi_{+}\right)-n_{F}\left(\xi_{-}\right)\right]}{\sqrt{\left|\vec{f}_{\mathbf{k}}\right|^{2}+\left|\vec{\xi}_{\mathbf{k}}^{-}\right|^{2}}},
$$

which is the van Vleck susceptibility.

[1] M. Smidman, M. B. Salamon, H. Q. Yuan, and D. F. Agterberg, Superconductivity and spin-orbit coupling in noncentrosymmetric materials: A review, Rep. Prog. Phys. 80, 036501 (2017).

[2] P. W. Anderson, Structure of "triplet" superconducting energy gaps, Phys. Rev. B 30, 4000 (1984).

[3] C.-K. Lu and S. Yip, Zero-energy vortex bound states in noncentrosymmetric superconductors, Phys. Rev. B 78, 132502 (2008).

[4] A. P. Schnyder and S. Ryu, Topological phases and surface flat bands in superconductors without inversion symmetry, Phys. Rev. B 84, 060504(R) (2011).

[5] M. H. Fischer, F. Loder, and M. Sigrist, Superconductivity and local noncentrosymmetricity in crystal lattices, Phys. Rev. B 84, 184533 (2011).

[6] M. Sigrist, D. F. Agterberg, M. H. Fischer, J. Goryo, F. Loder, S.-H. Rhim, D. Maruyama, Y. Yanase, T. Yoshida, and S. J. Youn, Superconductors with staggered non-centrosymmetricity, J. Phys. Soc. Jpn. 83, 061014 (2014).

[7] S. J. Youn, M. H. Fischer, S. H. Rhim, M. Sigrist, and D. F. Agterberg, Role of strong spin-orbit coupling in the superconductivity of the hexagonal pnictide srptas, Phys. Rev. B 85, 220505(R) (2012).

[8] K. Gotlieb, C.-Y. Lin, M. Serbyn, W. Zhang, C. L. Smallwood, C. Jozwiak, H. Eisaki, Z. Hussain, A. Vishwanath, and A. Lanzara, Revealing hidden spin-momentum locking in a high-temperature cuprate superconductor, Science 362, 1271 (2018).

[9] H. Shishido, T. Shibauchi, K. Yasu, T. Kato, H. Kontani, T. Terashima, and Y. Matsuda, Tuning the dimensionality of the heavy fermion compound $\mathrm{CeIn}_{3}$, Science 327, 980 (2010).

[10] Y. Mizukami, H. Shishido, T. Shibauchi, M. Shimozawa, S. Yasumoto, D. Watanabe, M. Yamashita, H. Ikeda, T. Terashima, H. Kontani, and Y. Matsuda, Extremely strong-coupling superconductivity in artificial two-dimensional kondo lattices, Nat. Phys. 7, 849 (2011).

[11] D. Maruyama, M. Sigrist, and Y. Yanase, Superconductivity without local inversion symmetry; Multi-layer systems, J. Phys.: Conf. Ser. 400, 022069 (2012).
[12] S. Khim, J. F. Landaeta, J. Banda, N. Bannor, M. Brando, P. M. R. Brydon, D. Hafner, R. Küchler, R. Cardoso-Gil, U. Stockert, A. P. Mackenzie, D. F. Agterberg, C. Geibel, and E. Hassinger, Field-induced transition from even to odd parity superconductivity in $\operatorname{cerh}_{2}$ as $_{2}$, arXiv:2101.09522.

[13] E. G. Schertenleib, M. H. Fischer, and M. Sigrist, Unusual $H$-T phase diagram of $\mathrm{CeRh}_{2} \mathrm{As}_{2}$ : The role of staggered non-centrosymmetricity, Phys. Rev. Research 3, 023179 (2021).

[14] D. Möckli and A. Ramires, Two scenarios for superconductivity in $\mathrm{CeRh}_{2} \mathrm{As}_{2}$, Phys. Rev. Research 3, 023204 (2021).

[15] A. Skurativska, T. Neupert, and M. H. Fischer, Atomic limit and inversion-symmetry indicators for topological superconductors, Phys. Rev. Research 2, 013064 (2020).

[16] S. Ono, H. C. Po, and H. Watanabe, Refined symmetry indicators for topological superconductors in all space groups, Sci. Adv. 6, eaaz8367 (2020).

[17] M. Geier, P. W. Brouwer, and L. Trifunovic, Symmetry-based indicators for topological Bogoliubov-de Gennes Hamiltonians, Phys. Rev. B 101, 245128 (2020).

[18] S.-J. Huang and Y.-T. Hsu, Faithful derivation of symmetry indicators: A case study for topological superconductors with time-reversal and inversion symmetries, Phys. Rev. Research 3, 013243 (2021).

[19] S. Ono, H. C. Po, and K. Shiozaki, $\mathbb{Z}_{2}$-enriched symmetry indicators for topological superconductors in the 1651 magnetic space groups, Phys. Rev. Research 3, 023086 (2021).

[20] P. A. Frigeri, D. F. Agterberg, A. Koga, and M. Sigrist, Superconductivity Without Inversion Symmetry: MnSi Versus $\mathrm{CePt}_{3} \mathrm{Si}$, Phys. Rev. Lett. 92, 097001 (2004).

[21] M. H. Fischer, Gap symmetry and stability analysis in the multiorbital fe-based superconductors, New J. Phys. 15, 073006 (2013).

[22] A. Ramires and M. Sigrist, Identifying detrimental effects for multiorbital superconductivity: Application to $\mathrm{Sr}_{2} \mathrm{RuO}_{4}$, Phys. Rev. B 94, 104501 (2016).

[23] A. Ramires, D. F. Agterberg, and M. Sigrist, Tailoring $T_{c}$ by symmetry principles: The concept of superconducting fitness, Phys. Rev. B 98, 024501 (2018).

[24] To lowest order, this would be $\left(d_{\mathbf{k}}^{\perp}\right)_{1}=\Delta^{\perp} \sin k_{z} \cos k_{z} / 2$ and $\left(d_{\mathbf{k}}^{\perp}\right)_{2}=\frac{t^{\prime}}{t} \Delta^{\perp} \sin k_{z} \sin k_{z} / 2$. 
[25] Note that exchange interactions can potentially give rise to interlayer pairing even at small $t_{z}$ [29].

[26] A. Abrikosov and L. P. Gor'kov, Spin-orbit interaction and the knight shift in superconductors, JETP 15, 752 (1962).

[27] T. Mizushima, Y. Tsutsumi, M. Sato, and K. Machida, Symmetry protected topological superfluid 3he-b, J. Phys.: Condens. Matter 27, 113203 (2015).
[28] A. J. Leggett, Spin Susceptibility of a Superfluid Fermi Liquid, Phys. Rev. Lett. 14, 536 (1965).

[29] H. Shimahara, Interlayer spin-singlet pairing induced by magnetic interactions in an antiferromagnetic superconductor, Phys. Rev. B 72, 134518 (2005). 